Disclosure of Interest: None declared

DOI: 10.1136/annrheumdis-2018-eular.5338

\section{AB1224 $\quad$ CT PET SCANS IN SUSPECTED LARGE VESSEL VASCULITIS AND GIANT CELL ARTERITIS - AN AUDIT IN THE BELFAST HEALTH AND SOCIAL CARE TRUST (BHSCT)}

\section{U.A. Laverty, E. Banks, M. McHenry. NHS, Belfast, Ireland}

Background: British Society of Rheumatology (BSR) guidelines, due to be updated in April 2018, recommend consideration of CT PET when there is suspicion of large-vessel GCA (LV-GCA) in patients with prominent systemic symptoms, limb claudication or persistently high-inflammatory markers despite adequate glucocorticosteroid therapy. Vascular Ultrasound is unhelpful in assess ment of the aorta.

Objectives: We investigated the use of CT PET in suspected cases of LV-GCA and its impact on management of patients in the BHSCT from August 2016 to August 2017

Methods: The IT support team in Royal Victoria Hospital provided a list of CTPET scans requested under the specific code for vasculitis and/or Pyrexia/infection and another code for general. Of the 250 scans identified under these codes, 34 scans were requested by Rheumatology for possible vasculitis following a review of the electronic care records. A proforma was used to aid data collection.

Results: Female:Male ratio was $3.25: 1$, with a mean age of $65.88 \%$ of the scans were requested due to a suspected diagnosis of vasculitis and $12 \%$ were for follow up of known vasculitis. $24 \%$ of CT PET scans were positive for large vessel vasculitis (LVV). The ESR was greater than $50 \mathrm{~mm} / \mathrm{hr}$ in $75 \%$ of positive scans. Of those patients with a positive CT PET scan, $88 \%$ were treated with steroids. Of those patients with a negative CT PET scan, $42 \%$ were treated with steroids. It is noteworthy that $29 \%$ of patients were on steroids at the time of CT PET which may impact results. $60 \%$ of patients who were on steroids at the time of CT PET were on $60 \mathrm{mg}$ of prednisolone daily. $31 \%$ of patients with negative scans were on steroids at the time of CT PET. $46 \%$ of patients with negative CT PET scans remained on steroid treatment. Steroid treatment was continued in patients with negative scans on basis of active aortic valve histology \pm clinical criteria for diagnosis of GCA \pm cerebral vasculitis on neuroimaging \pm polymyalgia rheumatica evident on CTPET. CT PET changed management in $65 \%$ of patients with positive results supporting steroid treatment and negative results guiding withdrawal of steroids.

Conclusions: We are fortunate to have access to CT PET in Northern Ireland. CT PET scans changed management in $65 \%$ of our patients, despite $29 \%$ of patients being on steroid treatment prior to CT PET. We wish to increase awareness of the role CTPET in the diagnosis and management of LVV. We are liaising with radiology colleagues to refine and maximise appropriate referrals for CT PET scans for patients with suspected vasculitis.

\section{REFERENCES:}

[1] BSR and BHPR guidelines for the management of giant cell arteritis. Bhaskar Dasgupta, et al. Rheumatology, Volume 49, Issue 8, 1 August 2010, Pages1594-1597, Published: 05 April 2010

[2] EULAR recommendations for the use of imaging in large vessel vasculitis in clinical practice. Christian Dejaco, et al. Ann Rheum Dis published online January 22, 2018

Disclosure of Interest: None declared DOI: 10.1136/annrheumdis-2018-eular.7390

\section{$\mathrm{AB} 1225$ \\ ANTIBODIES AGAINST HETEROGENEOUS NUCLEAR RIBONUCLEOPROTEINS (RA33) MAY HAVE A DIAGNOSTIC AND PROGNOSTIC VALUE IN RHEUMATOID ARTHRITIS, PARTICULARLY WHEN OTHER SEROLOGICAL TESTS ARE NEGATIVE}

\begin{abstract}
V.A. Aleksandrov ${ }^{1}$, L.N. Shilova ${ }^{2}$, A.V. Alexandrov ${ }^{1} .{ }^{1}$ Federal State Budgetary Science Institution Research Institute for clinical and experimental rheumatology ${ }^{2}$ The Department of Hospital Therapy, The Volgograd State Medical University, Volgograd, Russian Federation
\end{abstract}

Background: The high frequency of detection of antinuclear antibodies in rheumatoid arthritis (RA), although predominantly in low titres, allows us to consider the possibility of using varieties of these antibodies (primarily antibodies to RA-33) markers of RA especially in the early stages of the disease.

Objectives: To study the frequency of occurrence and determine the prognostic significance of antibodies to RA-33 in RA patients.

Methods: 57 RA patients were examined (mean age $50.5 \pm 10.1$ years)
The patients with the developed stage of the disease (47.4\%) prevailed, the average activity (DAS28 $=3.2-5.1$ ) of the pathological process $(86 \%)$; with the second radiographic stage (42\%) and functional class 2 (77\%).

The antinuclear factor was determined in the indirect immunofluorescence reaction on the HEp-2 cell line (norm <1:80), IgM-rheumatoid factor by the latex agglutination method (up to $20 \mathrm{IU} / \mathrm{ml}$ ), antibodies (norm up to $20 \mathrm{U} / \mathrm{ml}$ ) and antibodies of IgG class to RA33 antigen (norm up to $25 \mathrm{U} / \mathrm{ml}$ ) by ELISA test.

Results: ANF was detected in 18 patients with RA (32\%), and in $94.4 \%$ of cases (17 patients) a diagnostic titer of 1:80 and a diffuse type of glow of the nucleus were noted. The ANF titres did not correlate either with the activity of the disease or with extra-articular manifestations of RA $(p>0.1)$. Anti-RA33 was detected in 20 (35\%) RA patients: 18 positive people (90\%) had low positive anti-RA33 values (25 to $75 \mathrm{U} / \mathrm{ml}$ ). In 35 (61.4\%) of RA patients, anti-CCP was detected: 19 (33.3\%) had low positive values (20 to $60 \mathrm{U} / \mathrm{ml}), 16(28 \%)$ had highly positive values (more $60 \mathrm{U} / \mathrm{ml}$ ). IgM-RF was detected in $26(45.6 \%)$ patients in the values $>48 \mathrm{IU} / \mathrm{ml}$, as well as in $17(30 \%)$ patients with RA in the values from 24 to $48 \mathrm{IU} / \mathrm{ml} .14$ people $(54 \%)$ of patients with high IgM-RF levels had systemic manifestations of RA. It should be noted that anti-RA33 was detected in 9 patients with seropositive for anti-CCP and in 11 patients with seronegative both for anti-CCP and IgM-RF Thus, when the results of standard serological tests are negative, an additional study of anti-RA33 is recommended to diagnose seronegative RA.

When assessing the prognostic significance of the available clinical and laboratory and instrumental data we have analysed the results of the study of anti-CCP anti-RA33, as well as all data on the presence of $X$-ray erosions in patients with RA with magnetic resonance imaging or ultrasound examination of affected joints. The presence of erosion was noted in $23(40.4 \%)$ RA patients. The frequency of detection of anti-CCP in RA patients was significantly higher in the presence of erosive lesions of joints (19 of 35 patients were positive by anti-CCP, compared with 4 of 22 patients, negative for anti-CCP, $\chi 2=5.89, p=0.015)$. In the RA group of patients positive for a wide range of antibodies (IgM-RF, anti-CCP, anti-RA33) the signs of joint erosion were identified in 22 of 46 patients, and in isolated increase only anti-RA33 - in 1 patient out of 11 people in this group $\chi 2=4.04$ $\mathrm{p}=0.044)$.

Conclusions: In the presence of highly positive anti-CCP values, RA patients have a more unfavourable prognosis, while an isolated increase in anti-RA33 is associated with a "milder progression" of the disease and inversely proportional to erosive processes in the joints.

Disclosure of Interest: None declared

DOI: 10.1136/annrheumdis-2018-eular.6309

\section{$\mathrm{AB} 1226$ \\ THE DIAGNOSTIC VALUE OF SERUM KL-6 IN CONNECTIVE TISSUE DISEASE ASSOCIATED INTERSTITIAL LUNG DISEASE}

X. Wu, C.N. Luo, Y.M. Shi, L.J. Wu. Department of Rheumatology and Immunology, People's Hospital of Xinjiang Uygur Autonomous Region, Urumqi, China

Background: The connective tissue diseases is a group of inflammatory, immune-mediated diseases.CTD often leads to autoimmunity and subsequent tissue injury. It is an important contribute to thoracic changes, particularly interstitial lung disease, is are the main causes of mortality and morbidity among patients with connective tissue diseases. Prognosis and response to therapy are the most pressing challenges for connective tissue disease-associated ILD (CTD-ILD). At present, the basic methods for the diagnosis of various types of ILD includes high-resolution computed tomography, bronchoscopy, and surgical lung biopsy. In addition, continuous lung function tests are commonly used to monitor disease activity and predict the outcome of patients with ILDs, But these tests require specific inspection machines and Repeatability is not good. At present, many biomarkers have been developed to detect ILDs, and the most important biomarkers is $\mathrm{KL}-6$ and lung surface active protein $A(S P-A)$ and surfactant protein $D(S P-D)$ witch secreted by alveolar epithelial type II cells. But Relevant studies have shown that the sensitivity, specificity and accuracy of KL-6 are higher than SP-A and SPD.

Objectives: To evaluate the diagnosis of the serum Krebs von den Lungen- 6 (KL-6) for the interstitial lung disease(ILD) associated with connective tissue diseases(CTD).

Methods: We retrospectively analysed the medical records of 50 patients with CTD associated ILD,46 CTD patients without ILD. Measurement of serum KL-6 levels and pulmonary function tests performed in parallel were reviewed.T test, $\mathrm{X}^{2}$ test, non-parametric test, SPSS and correlation analysis were used for data analysis

Results: The significantly higher levels of KL-6 were determined in the CTD-ILD group than in either the CTD without pulmonary involvement group $(\mathrm{P}<0.05)$ Serum KL-6 correlated negatively with forced vital capacity(FVC\%) (\%predicted) forced expiratory volume in one second (FEV1)(\%predicted) and diffusing capacity of the lung for carbon monoxide (DLCO) (\% predicted). By the ROC curves of serum KL-6 levels in 96 patients. The optimal cutoff value of serum KL- 6 
for a diagnosis of CTD-ILD was $500 \mathrm{mg} / \mathrm{L}$, and the sensitivity and specificity were $82.0 \%$ and $89.1 \%$, respectively. The area under the curve (AUC) was 0.877 . By multivariate analysis, we found only the reduced DLCOSB\%, and elevated serum KL-6

Abstract AB1226 - Table 1. single factor and multifactor analysis of the risk factors of ILD

\begin{tabular}{|c|c|c|c|}
\hline \multirow[t]{2}{*}{ Variables } & Univariate analysis & \multicolumn{2}{|c|}{ Multivariate regression analysis } \\
\hline & 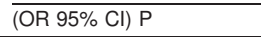 & (OR 95\% C & \\
\hline Age & $0.956(0.924,0.988) 0.008$ & & \\
\hline VC\% & $1.051(1.018,1.084) 0.002$ & & \\
\hline FVC \% & $1.030(1.004,1.057) 0.022$ & & \\
\hline FEV1\% & $1.032(1.0041 .062) 0.026$ & & \\
\hline TLcoSB $\%$ & $1.081(1.042,1.120) 0.000$ & $1.097(1.031,1.168)$ & 0.004 \\
\hline KL-6 & $0.998(0.997,0.999) 0.000$ & $0.997(0.996,0.999)$ & .000 \\
\hline
\end{tabular}

Conclusions: The serum KL-6 is a valuable biomarker for CTD-ILD diagnosis and even as a predictive factor could be used to identify the clinical development of ILD.

Disclosure of Interest: None declared

DOI: 10.1136/annrheumdis-2018-eular.6316

\section{AB1227 INFLAMMATORY FINDINGS ON ULTRASOUND AND MR CAN PREDICT FUTURE DEVELOPMENT OF RHEUMATOID ARTHRITIS IN PATIENTS WITH SERONEGATIVE, UNDIFFERENCIATED ARTHRITIS}

Y. Kondo ${ }^{1,2}$, Y. Kaneko ${ }^{2}$, Y. Inoue ${ }^{2}$, C. Takahashi ${ }^{2}$, K. Sakata ${ }^{2}$, K. Yamaoka ${ }^{2}$, S. Sato ${ }^{1}$, T. Takeuchi ${ }^{2}$. ${ }^{1}$ Department of Internal Medicine, Division of Rheumatology, Tokai Unversity School of Medicine, Kanagawa; ${ }^{2}$ Department of Internal Medicine, Division of Rheumatology, Keio University School of Medicine, Shinjuku, Tokyo, Japan

Background: The 2010 rheumatoid arthritis (RA) classification criteria has been verified to classify patients early as having RA more efficiently than the 1987 criteria. However, sensitivity of this criteria decreased remarkably in patients whose rheumatoid factor (RF) and anticitrullinated antibodies (ACPA) were both negative. Modern imaging technique including ultrasound (US) and magnetic resonance imaging (MRI) are more sensitive than physical examination for detecting joint inflammation objectively, however, US may offer only slight additional value when assessing patients with positive ACPA and RF. Reliability and value of inflammatory findings detected by US and MRI in seronegative, undifferentiated arthritis (UA) patients are still unclear.

Objectives: To clarify benefits of US and MRI for predicting futuristic diagnosis of RA in UA patients with neither ACPA nor RF.

Methods: Consecutive, untreated, early arthritis patients who underwent both US and contrast enhanced MRI of 22 sites including bilateral wrists, MCP and PIP joints were enrolled. Synovitis and tenosynovitis were defined as inflammatory findings of US and MRI. Concordance between swollen joint counts (SJC) by experienced physician, inflammatory findings of US and MRI were assessed. We defined UA as non-fulfilment of the 2010 RA classification criteria and the clinical diagnosis pf RA as the initiation of disease modifying anti-rheumatic drugs.

Results: Seventy one patients were included in the analysis. Fifty eight (82\%) were female, the median age was 63 years old, and the mean symptom duration 3 months. Forty eight $(67.6 \%)$ did not fulfil the 2010 criteria being defined as UA, among which thirty six were seronegative. In all patients, the concordance of detecting inflammation was quite high between MRI and US (к: 0.67-0.70). In 36 seronegative UA patients, SJC detected by physical examinations were fewer than US or MRI (2.2 vs 3.9, $\mathrm{p}<0.05)$ and discrepancy between clinical SJC and inflammatory findings by US/MRI were more frequent in seronegative UA patients

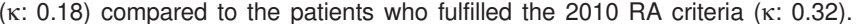
Although only 4 of the seronegative UA patients would have fulfilled the 2010 criteria if inflammation findings by US/MRI were used instead of physical examinations, 21 seronegative UA were diagnosed with clinical RA (sensitivity 19\% and specificity $100 \%$ ). One or more inflammatory arthritis in wrists and MCP joints detected by US or MRI in seronegative patients significantly predicted the development of RA with a good sensitivity of $62 \%$ and specificity of $87 \%$ (Odds ratio, 10.5).

Conclusions: Our study suggested that both US and MRI inflammation findings are reliable with a good concordance and can be useful as predictor for futuristic development of RA in UA patients without ACPA and RF.

REFERENCES:

[1] Kaneko Y, et al. Rheumatology (Oxford). 2011 Jul;50(7):1268-74.

[2] Zufferey P, et al. Joint Bone Spine. 84(3): 299-303, 2017

[3] Kawashiri S, et al. Mod Rheumatol (2013) 23:36-43

[4] Hirata A, et al. Arthritis Care Res (Hoboken). 2017 Jun;69(6):801-806
Disclosure of Interest: None declared

DOI: 10.1136/annrheumdis-2018-eular.4147

\section{$\mathrm{AB} 1228$ \\ SONOELASTOGRAPHY OF QUADRICEPS IS MORE ACCURACY THAN GRIP STRENGTH TO PREDICT LOW MUSCLE MASS}

Y.-C. Chen. Rheumatology, KAOHSIUNG CHANG GUNG MEMORIAL

HOSPITAL, Kaohsiung, Taiwan, Province of China

Background: Reduced muscle mass had associated with higher mortality. So it is mandatory for simple techniques to early detection sof arcopenia. However, some patients with low grip strength had normal muscle mass, especially those with rheumatoid arthritis.

Objectives: Our objective was to examine the validity of sonoelastography to predict sarcopenia in osteoporotic patients.

Methods: We conducted an observational study in Kaohsiung Chang Gang Memorial Hospital. Low muscle mass was determined using a dual-energy X-ray absorptiometry. Sonoelastography was performed over mid thigh over quadriceps muscle. We measure grip strength and hardness and elastography ratio of quadriceps over subcutaneous fat tissue. Logistical regression was used to find the factors to predict low muscle mass. While ROC analysis was used to find best cut-off point.

Results: A total 122 (68 low muscle mass, 54 normal muscle mass) patients were enrolled. The mean age was $79.26 \pm 6.77$ years in low muscle mass group and $74.7 \pm 13.49$ in normal muscle mass group $(p=0.017)$. Most patients $(86.9 \%)$ were women. Sonoelastography showed low muscle mass patients had more soft than normal muscle mass patients, furthermore the elastography ratio of quadriceps over subcutaneous tissue was lower than normal muscle mass patients. Using logistical regression, grip strength, sex and age cannot be used to predict low muscle mass, while the hardness and ratio had statistically significant to predict low muscle mass $(\mathrm{p}=0.001)$. When the cut points determined by receiver operating characteristic (ROC) curve analysis were applied, The best cut-point of hard ness was $64.79 \%$ (sensitivity, $0.741 ; 1$-specificity, 0.147 ), while the best cut-point of quadriceps over subcutaneous tissue was 0.81 (sensitivity, $0.815 ; 1$-specificity, $0.118)$.

Abstract AB1228 - Table 1

\begin{tabular}{lcccc}
\hline & $\begin{array}{c}\text { Regression } \\
\text { coefficient }\end{array}$ & S.E. & $\begin{array}{c}P \\
\text { value }\end{array}$ & OR \\
\hline Gender & 1.976 & 1.063 & 0.063 & $\begin{array}{c}7.216(0.899- \\
57.939)\end{array}$ \\
Age & 0.043 & 0.041 & 0.297 & $1.043(0.963-1.132)$ \\
Hardness & -4.015 & 0.897 & 0.001 & $0.018(0.003-0.105)$ \\
Ratio & -3.927 & 0.856 & 0.001 & $0.019(0.004-0.105)$ \\
Grip & 0.218 & 0.640 & 0.734 & $1.243(0.355-4.356)$ \\
strength & & & & \\
\hline
\end{tabular}

Conclusions: Our findings indicate that sonoelastography is more accurate to predict low muscle mass. The measurement include quadriceps over subcutaneous tissue and hardness of the quadriceps. Although, grip strength is less expensive for evaluation of muscular weakness, from this findings, it is not a reliable method for evaluation of low muscle mass. The possible reason is that sarcopenia initial occur at thigh muscle and then spread up to upper limb. So grip strength is not a accurate method to screen sarcopenia.

\section{REFERENCE:}

[1] Cruz-Jentoft AJ, Baeyens JP, Bauer JM, Boirie Y, Cederholm T, Landi F, Martin FC, Michel JP, Rolland Y, Schneider SM, et al: Sarcopenia: European consensus on definition and diagnosis: Report of the European Working Group on Sarcopenia in Older People. Age and ageing 2010, 39 (4):412-423.

Acknowledgements: This study was supported by Kaohsiung Chang Gung Memorial Hospital and Chang Gung University College of Medicine, Kaohsiung grant CMRP

Disclosure of Interest: None declared

DOI: 10.1136/annrheumdis-2018-eular. 4145 